\title{
Friabilidad y su relación con algunas propiedades físicas y químicas de suelos cultivados con caña de azúcar
}

\author{
Friability and its relationship with some physical and chemical properties \\ of soils under sugar cane
}

Edgar Alvaro Avila P. ${ }^{1}$; Fabio Rodrigo Leiva B. ${ }^{2}$; Aquiles Enrique Darghan C. ${ }^{3}$

\footnotetext{
${ }^{1}$ Docente. Ph. D. Universidad del Tolima. Ibagué, Colombia, eaavila@ut.edu.co.

${ }^{2}$ Docente. Ph. D. Universidad Nacional de Colombia. Bogotá D.C., Colombia, frleivab@unal.edu.co.

${ }^{3}$ Docente. Ph. D. Universidad Nacional de Colombia. Bogotá D.C., Colombia, aqedarghanco@unal.edu.co.
}

Citar: Avila, E.; Leiva, F.; Darghan, A. 2017. Friabilidad y su relación con algunas propiedades físicas y químicas de suelos cultivados con caña. Rev. Cienc. Agr. 34(2): 19 - 32. doi: http://dx.doi.org/10.22267/rcia.173402.69.

Recibido: Marzo 29 de $2017 . \quad$ Aceptado: Agosto 23 de 2017.

\section{RESUMEN}

La friabilidad es un indicador importante de la calidad física de los suelos y su comportamiento está ligado a la variabilidad de otras propiedades edáficas. El objetivo de esta investigación fue evaluar la relación de la friabilidad con diferentes propiedades físicas y químicas de suelos cultivados con caña de azúcar. El muestreo de suelos se realizó en los dos primeros horizontes (Ap y A1) de 91 sitios correspondientes a 5 órdenes de suelos (Inceptisol, Molisol, Vertisol, Alfisol y Ultisol). Se seleccionaron 10 agregados por cada uno de los cuatro rangos de tamaño $(2,0$ a 4,79; 4,80 a 9,49; 9,50 a 19,00 y 19,01 a 35,70 mm) que fueron los predominantes en los 182 horizontes. Los 7280 agregados fueron secados en invernadero y horno y posteriormente sometidos a compresión para determinar la resistencia al rompimiento (RR) y el índice de friabilidad correspondiente al límite inferior $\left(\mathrm{IF}_{\mathrm{i}}\right)$ del intervalo de confianza del coeficiente de variación. Se determinó el diámetro ponderado medio (DPM), el estado de agregación (Ea), la densidad aparente (Da), la porosidad total (Pt), el pH y la conductividad eléctrica (CE) de las muestras de suelos. Se utilizaron medidas de tendencia central y dispersión, gráficos de superficies tridimensionales y un modelo lineal para un Diseño Completamente al Azar en arreglo factorial incompleto. No se encontró efecto del DPM, 
la Da, el pH y la CE sobre el $\mathrm{IF}_{\mathrm{i}}$, pero sí del Ea y la Pt. Los valores del $\mathrm{IF}_{\mathrm{i}}$ se incrementaron a mayor porosidad total y a mayor estado de agregación.

Palabras clave: índice de friabilidad, estabilidad de agregados, densidad aparente, porosidad total, $\mathrm{pH}$.

\begin{abstract}
Friability is an important indicator of the physical quality of soils and it is linked to the variability of other edaphic properties. This study aimed to evaluate the relationship between friability and different physical and chemical properties of soils cultivated with sugar cane. The sampling was carried out in the first two horizons (Ap and A1) of 91 sites corresponding to 5 soil orders (Inceptisol, Molisol, Vertisol, Alfisol and Ultisol). Ten aggregates were selected for each of the four size ranges (2.0 to $4.79,4.80$ to $9.49,9.50$ to 19.00 and 19.01 to $35.70 \mathrm{~mm}$ ) that predominated in the 182 horizons. The 7,280 aggregates were dried in a greenhouse and oven and, subsequently, subjected to compression to determine the compressive strength (CS) and the friability index that corresponded to the lower limit $\left(\mathrm{FI}_{\mathrm{i}}\right)$ of the confidence interval of the coefficient of variation . It was determined the mean weight diameter (WMD), the aggregation state (AS), the bulk density (AD), the total porosity (TP), the $\mathrm{pH}$ and the electrical conductivity (EC) of the soil samples. In addition, measurements of dispersion and central tendency, three-dimensional surface graphs, and a linear model were used with a completely random design using an incomplete factorial arrangement. No effect was seen from the WMD, AD, $\mathrm{pH}$, or EC on the $\mathrm{FI}_{\mathrm{i}}$; however, the AS and the TP had significant effects from the inferential point of view.
\end{abstract}

Keywords: friability index, aggregate stability, bulk density, total porosity, $\mathrm{pH}$.

\section{INTRODUCCIÓN}

La friabilidad entendida como la tendencia de una masa de suelo no confinada a romperse y desmoronarse bajo un esfuerzo aplicado en un rango particular de tamaño de agregados más pequeños (Munkholm, 2011; Reis et al., 2014). Es una propiedad que no solo es importante en los suelos sometidos a labranza convencional, sino que también es de crucial importancia en el establecimiento de cultivos bajo siembra directa (Macks et al., 1996). La friabilidad del suelo es un factor importante en la determinación de su posible respuesta a la labranza. Un suelo friable requiere un mínimo de labranza para producir una ade- cuada cama de siembra con agregados estables pequeños que proporcionan condiciones óptimas para el crecimiento de la planta; por el contrario, un suelo no friable puede demandar alta energía en las operaciones de labranza que generan impactos negativos en el ambiente (Esengun et al., 2007; Sefeedpari et al., 2014), y finalmente producir una pobre cama de siembra, con agregados muy finos que pueden conducir a sellamiento y encostramiento superficial en presencia de lluvia o riego, o terrones grandes que proporcionan condiciones inadecuadas para la germinación, emergencia y establecimiento de las plantas (Munkholm, 2011). 
La friabilidad es una propiedad física cuyo comportamiento se ve influenciado por diferentes propiedades o características del suelo, entre ellas: el contenido de agua, la estabilidad de los agregados, la densidad aparente (Ye et al., 2017), la porosidad (Munkholm et al., 2002a y b; Munkholm et al., 2012), la microestructura (Dexter y Watts, 2001), la materia orgánica (Munkholm 2011; Schjǿnning et al., 2012), la fracción arcilla (Schjǿnning et al., 2012) y la concentración y composición de la solución del suelo (Rahimi et al., 2000).

La estabilidad estructural y friabilidad del suelo están relacionadas con la fractura por fragilidad del suelo (Braunack et al., 1979; Dexter y Watts, 2001). El término estabilidad se utiliza para describir la capacidad que tiene el suelo de mantener una estructura coherente en presencia de agua libre; sin embargo, se conocen pocos trabajos en los que se relacione el efecto de la estabilidad estructural del suelo, medida a partir del diámetro ponderado medio (DPM) y el estado de agregación (Ea), con el índice de friabilidad de los suelos. La friabilidad depende en gran medida de la facilidad de fractura de los agregados del suelo y el desarrollo progresivo de planos de fractura que originan grietas, y con estas, la pérdida de resistencia de los mismos (Munkholm et al., 2012). La propagación de las grietas en un suelo no confinado sometido a presión depende de la resistencia de los extremos de las fisuras o microgrietas y la abundancia y morfología (orientación y conectividad) de los poros (Munkholm et al., 2016). Un fuerte vínculo entre la friabilidad y fragmentación del suelo con el espacio poroso ocupado por aire fue reportado por Munkholm et al. (2002a). En un trabajo posterior, Munkholm et al. (2012) confirmaron la correlación significativa entre la porosidad y la friabilidad en suelos de Canadá mediante la observación de la estructura del suelo en imágenes tridimensionales. Para Alaoui et al. (2011), la alta proporción de microporos, junto con un alto número de puntos de contacto entre las partículas del suelo, conduce a una mayor resistencia interna a la ruptura en los agregados.

En las tierras de cultivo, el uso de maquinaria agrícola en las actividades de labranza es una de las causas más comúnmente asociada a la reducción de la porosidad y al incremento de la densidad aparente (Etana et al., 2013; Kuncoro et al., 2014). De acuerdo con Munkholm et al. (2002a y b), en general, la resistencia al rompimiento (RR) se incrementa con el aumento de la densidad aparente (Larionov et al., 2017) y consecuentemente la friabilidad disminuye. Así mismo, Macks et al. (1996), encontraron que los suelos con baja friabilidad tienden a presentar alta densidad aparente, requiriendo un esfuerzo mayor en la labranza para producir una adecuada cama de siembra. Según estos autores, suelos con estas características son considerados inadecuados para la siembra directa. Algunos investigadores han encontrado también relación entre la friabilidad y algunas propiedades químicas de los suelos; en este sentido, Schjǿnning et al. (2012), Rahimi et al. (2000) y Munkholm (2011), sugieren que la RR y por lo tanto la friabilidad dependen de la cantidad de arcilla dispensable, tipo y tamaño de arcilla, contenido de materia orgánica y la concentración y composición de la solución del suelo.

El objetivo de la presente investigación fue evaluar la relación de la friabilidad con diferentes propiedades físicas y químicas de los suelos bajo cultivo de caña de azúcar en algunos sectores del valle geográfico del Río Cauca (Colombia). 


\section{MATERIALES Y MÉTODOS}

Sitios de muestreo. La investigación se llevó a cabo en fincas dedicadas al cultivo de caña de azúcar con prácticas similares de manejo mecanizado de los suelos, localizadas en el centro y sur del departamento del Valle del Cauca y norte del departamento del Cauca, Colombia (Tabla 1).

El manejo de los suelos en las fincas señaladas comprende un primer pase de arado para destruir e incorporar residuos de cosecha, un posterior pase de rastra para reducir el tamaño de los terrones del suelo y finalmente un surcado para dejar el lote listo para la siembra. La siembra se realiza de manera manual, para ello se utilizan los esquejes o trozos de tallo de aproximadamente $60 \mathrm{~cm}$ de longitud. El ciclo de desarrollo del cultivo es de aproximadamente 12 meses hasta la cosecha, periodo durante el cual, en las fincas referidas, no se realiza mecanización del suelo. La cosecha del cultivo se realiza de forma mecanizada, pero antes de esta labor se somete el cultivo a quema controlada para reducir el volumen de hojas.

Los predios hacen parte del bosque seco tropical (IGAC, 1977) y de la unidad climática correspondiente al Tropical Lluvioso de Sabana (Awi) (clasificación climática de Köppen). Los suelos estudiados pertenecen a los órdenes Inceptisol, Vertisol, Molisol, Alfisol y Ultisol (USDA, 2010) y se localizan en los planos de terraza de la llanura aluvial del Río Cauca. Las principales propiedades y características físicas y químicas de estos suelos se encuentran en la Tabla 1.

A partir del estudio detallado de suelos de la zona donde se localizaron los predios (IGAC y CENICAÑA, 2006), en cada uno de ellos se localizó un perfil modal de suelo y dentro de los límites del mismo se ubicaron cinco sitios de observación distantes entre sí aproximadamente $100 \mathrm{~m}$. En cada sitio de observación se realizó la delimitación y muestreo de los dos primeros horizontes (Ap y A1), en cajuelas de aproximadamente $50 \mathrm{x}$ $50 \mathrm{~cm}$ de lado y $50 \mathrm{~cm}$ de profundidad.

Toma y preparación de muestras. En los cinco sitios de muestreo localizados en cada una de las 18 fincas (excepto en la primera finca donde se localizaron 6 sitios) se muestrearon los dos primeros horizontes del suelo (Ap y A1), de cada horizonte se tomó un bloque de aproximadamente $30 \mathrm{~cm}$ de largo x $18 \mathrm{~cm}$ de ancho y $12 \mathrm{~cm}$ de espesor, de tal forma que en total se colectaron 182 bloques. Para evitar el deterioro o alteración de los bloques, estos se envolvieron en plástico adherente y posteriormente, se transportaron en cajas de madera hasta el laboratorio donde se separaron manualmente los agregados de cada bloque aprovechando los planos de debilidad natural del suelo. Estos agregados se pasaron a través de un conjunto de tamices dispuestos en secuencia vertical desde el tamaño de malla de $50 \mathrm{~mm}$ (tamiz superior) hasta el tamaño de malla de $1 \mathrm{~mm}$ (tamiz inferior), lo que permitió seleccionar 4 rangos de tamaño de agregados dominantes: 2,0 a $4,79 \mathrm{~mm}, 4,80$ a $9,49 \mathrm{~mm}, 9,50$ a $19,00 \mathrm{~mm}$ y 19,01 a $35,70 \mathrm{~mm}$. De cada rango de tamaño se tomaron aleatoriamente diez agregados por muestra, para un total de 7280 agregados que fueron secados durante cinco días en invernadero a una temperatura promedio diurna de $29^{\circ} \mathrm{C}$. Posteriormente se llevaron al horno a una temperatura constante de $40^{\circ} \mathrm{C}$ durante 48 horas (Guimarães et al., 2009). Con el propósito de evitar que las muestras ganaran humedad del ambiente luego del secado al horno, los agregados se embolsaron y se depositaron en un recipiente de cierre hermético hasta el momento en que se sometieron a pesaje y posterior ensayo de compresión. 
Tabla 1. Localización geográfica de las fincas donde se realizó el muestreo y principales propiedades físicas y químicas de los suelos.

\begin{tabular}{|c|c|c|c|c|c|c|c|c|c|}
\hline $\begin{array}{l}\text { Finca } \\
\text { No. }\end{array}$ & $\begin{array}{c}\text { Coordenadas } \\
\text { sitio de muestreo } \\
\text { central }\end{array}$ & $\begin{array}{c}\text { Taxonomía de los } \\
\text { suelos }\end{array}$ & $\begin{array}{l}\text { Número de } \\
\text { sitios de } \\
\text { muestreo }\end{array}$ & $\begin{array}{c}\text { DPM } \\
\text { (mm) } \\
\text { pond. } \\
\text { (Ap/ } \\
\text { A1) }\end{array}$ & $\begin{array}{c}\text { Ea } \\
\text { (\%) } \\
\text { pond. } \\
\text { (Ap/ } \\
\text { A1) }\end{array}$ & $\begin{array}{c}\text { Da } \\
\text { (Mg } \\
\left.\text { m }^{-3}\right) \\
\text { pond. } \\
\text { (Ap/ } \\
\text { A1) }\end{array}$ & $\begin{array}{c}\mathrm{Pt} \\
\text { pond. } \\
\text { (\%) } \\
\text { (Ap/ } \\
\text { A1) }\end{array}$ & $\begin{array}{l}\mathrm{pH} \\
\text { pond. } \\
\text { (Ap/ } \\
\text { A1) }\end{array}$ & $\begin{array}{c}\mathrm{CE} \\
(\mathrm{mS} \\
\left.\mathrm{cm}^{-1}\right) \\
\text { pond. } \\
\text { (Ap/ } \\
\text { A1) }\end{array}$ \\
\hline \multirow{2}{*}{1} & $3^{\circ} 17^{\prime} 32.9^{\prime \prime} /$ & \multirow{2}{*}{ Udertic Haplustalfs } & \multirow{2}{*}{6} & 2,90 & 81,70 & 1,20 & 52,70 & 5,60 & 0,40 \\
\hline & $76^{\circ} 30^{\prime} 39.2^{\prime \prime}$ & & & 3,00 & 82,90 & 1,30 & 49,30 & 5,70 & 0.30 \\
\hline \multirow{2}{*}{2} & $3^{\circ} 16^{\prime} 03.4^{\prime \prime} /$ & \multirow{2}{*}{ Vertic Endoaqualfs } & \multirow{2}{*}{5} & 3,30 & 78,30 & 1,30 & 49,50 & 5,40 & 1,10 \\
\hline & $76^{\circ} 30^{\prime} 20.2^{\prime \prime}$ & & & 3,00 & 76,40 & 1,30 & 49,90 & 5,90 & 0,50 \\
\hline \multirow{2}{*}{3} & $3^{\circ} 37^{\prime} 31.6^{\prime \prime} /$ & \multirow{2}{*}{ Vertic Haplustolls } & \multirow{2}{*}{5} & 1,30 & 46,20 & 1,40 & 44,90 & 7,80 & 1,10 \\
\hline & $76^{\circ} 19^{\prime} 50.2^{\prime \prime}$ & & & 1,30 & 45,80 & 1,40 & 45,30 & 7,80 & 1,20 \\
\hline \multirow{2}{*}{4} & $3^{\circ} 46^{\prime} 52.1^{\prime \prime} /$ & \multirow{2}{*}{ Typic Calciusterts } & \multirow{2}{*}{5} & 1,50 & 57,20 & 1,30 & 44,20 & 8,10 & 1,60 \\
\hline & $76^{\circ} 22^{\prime} 20.1^{\prime \prime}$ & & & 1,20 & 52,10 & 1,30 & 46,90 & 8,40 & 1,30 \\
\hline \multirow{2}{*}{5} & $3^{\circ} 7^{\prime} 42.5^{\prime \prime} /$ & \multirow{2}{*}{ Typic Palehumults } & \multirow{2}{*}{5} & 3,00 & 80,30 & 1,40 & 43,80 & 4,80 & 0,30 \\
\hline & $76^{\circ} 24^{\prime} 42.2^{\prime \prime}$ & & & 2,50 & 75,50 & 1,40 & 42,20 & 4,70 & 0,30 \\
\hline \multirow{2}{*}{6} & $3^{\circ} 5^{\prime} 30.4 " /$ & \multirow{2}{*}{ Typic Palehumults } & \multirow{2}{*}{5} & 3,20 & 83,10 & 1,40 & 42,00 & 4,90 & 0,40 \\
\hline & $76^{\circ} 28^{\prime} 03.2^{\prime \prime}$ & & & 3,10 & 79,90 & 1,40 & 42,80 & 5,30 & 0,40 \\
\hline \multirow{2}{*}{7} & $3^{\circ} 4^{\prime} 4.5^{\prime \prime}$ & \multirow{2}{*}{ Vertic Haplustalfs } & \multirow{2}{*}{5} & 3,10 & 76,80 & 1,40 & 42,00 & 5,80 & 0,60 \\
\hline & $76^{\circ} 25^{\prime} 54.9^{\prime \prime}$ & & & 3,80 & 86,00 & 1,50 & 41,50 & 5,90 & 0,80 \\
\hline \multirow{2}{*}{8} & $3^{\circ} 13^{\prime} 6.2^{\prime \prime} /$ & \multirow{2}{*}{ Udertic Haplustalfs } & \multirow{2}{*}{5} & 4,20 & 92,00 & 1,50 & 40,80 & 5,10 & 0,30 \\
\hline & $76^{\circ} 30^{\prime} 23.1^{\prime \prime}$ & & & 4,60 & 91,00 & 1,50 & 40,40 & 5,10 & 0,60 \\
\hline \multirow{2}{*}{9} & $3^{\circ} 38^{\prime} 11.5^{\prime \prime} /$ & \multirow{2}{*}{ Typic Haplustolls } & 5 & 1,30 & 41,60 & 1,30 & 47,40 & 7,90 & 1,80 \\
\hline & $76^{\circ} 18^{\prime} 23.4^{\prime \prime}$ & & 3 & 1,40 & 47,60 & 1,40 & 44,50 & 7,40 & 1,60 \\
\hline 10 & $3^{\circ} 40^{\prime} 12^{\prime \prime} /$ & Tynic Hanluctantc & 5 & 0,50 & 18,70 & 1,30 & 51,20 & 8,10 & 2,60 \\
\hline 10 & $76^{\circ} 24^{\prime} 31^{\prime \prime}$ & 1уріс паріustepts & 5 & 0,40 & 12,90 & 1,50 & 46,30 & 8,20 & 1,50 \\
\hline 11 & $3^{\circ} 47^{\prime} 45.2^{\prime \prime} /$ & Vertic Hanlustolls & 5 & 2,60 & 76,40 & 1,30 & 49,10 & 6,80 & 0,90 \\
\hline 11 & $76^{\circ} 17^{\prime} 19.6^{\prime \prime}$ & vertic Haplustolls & 5 & 2,20 & 71,70 & 1,30 & 46,10 & 6,70 & 0,70 \\
\hline & $3^{\circ} 42^{\prime} 24.7^{\prime \prime} /$ & Petrocalcic & 5 & 2,20 & 67,10 & 1,50 & 38,70 & 7,00 & 1,30 \\
\hline & $76^{\circ} 23^{\prime} 8^{\prime \prime}$ & Haplusterts & 5 & 1,80 & 60,40 & 1,50 & 39,20 & 7,40 & 1,00 \\
\hline 13 & $3^{\circ} 55^{\prime} 35.8^{\prime \prime} /$ & Fntic Hanlusterts & 5 & 1,60 & 70,50 & 1,20 & 49,60 & 5,90 & 0,80 \\
\hline 13 & $76^{\circ} 18^{\prime} 26.8^{\prime \prime}$ & Entic Haplusterts & 5 & 1,30 & 67,00 & 1,20 & 47,40 & 6,30 & 0,60 \\
\hline 14 & $3^{\circ} 42^{\prime} 6.11^{\prime \prime} /$ & Fntic Hanluctolls & 5 & 2,70 & 70,10 & 1,60 & 39,20 & 6,30 & 0,70 \\
\hline & $76^{\circ} 17^{\prime} 49.1^{\prime \prime}$ & Entıc Haplustolls & 5 & 1,90 & 61,90 & 1,50 & 41,30 & 6,50 & 0,60 \\
\hline 15 & $4^{\circ} 4^{\prime} 38.3^{\prime \prime} /$ & Fntic Hanlusterts & 5 & 0,60 & 28,60 & 1,50 & 42,00 & 5,70 & 0,50 \\
\hline & $76^{\circ} 14^{\prime} 12.5^{\prime \prime}$ & & & 0,60 & 30,80 & 1,50 & 40,60 & 5,80 & 0,90 \\
\hline 16 & $4^{\circ} 4^{\prime} 59^{\prime \prime} /$ & Typic Endoaquerts & 5 & 0,60 & 32,60 & 1,30 & 47,80 & 6,60 & 0,90 \\
\hline 10 & $76^{\circ} 17^{\prime} 16^{\prime \prime}$ & & 5 & 0,60 & 31,20 & 1,30 & 45,70 & 6,60 & 0,90 \\
\hline 17 & $3^{\circ} 31^{\prime} 37.8^{\prime \prime} /$ & Fntic Hanlustolls & 5 & 0,50 & 18,70 & 1,20 & 54,40 & 5,90 & 1,80 \\
\hline 17 & $76^{\circ} 28^{\prime} 21.7^{\prime \prime}$ & Entıc наріustolls & 5 & 0,50 & 18,60 & 1,30 & 51,10 & 6,00 & 1,80 \\
\hline 18 & $3^{\circ} 3^{\prime} 1^{\prime \prime} /$ & Tynic Palehumults & 5 & 1,70 & 57,00 & 1,30 & 49,30 & 5,20 & 0,40 \\
\hline 10 & $76^{\circ} 30^{\prime} 43.2^{\prime \prime}$ & iурic r dientumints & $\mathrm{J}$ & 1,80 & 58,00 & 1,30 & 47,10 & 5,00 & 0,30 \\
\hline
\end{tabular}

DPM: diámetro ponderado medio; Ea: estado de agregación; Da: densidad aparente; Pt: porosidad tota; pH: reacción;

CE: conductividad eléctrica. Pond.: valor ponderado de la propiedad. Sitios mues.: sitio de muestreo de suelos. 
De los agregados inicialmente separados de cada bloque de suelo se obtuvo también las muestras para las determinaciones del DPM y el Ea (tamices de tamaño de malla 6,3; 4,0; 2,$0 ; 1,0$ y 0,425mm). Para la determinación de la $\mathrm{Da}$, en cada horizonte se recolectaron por duplicado muestras no disturbadas en cilindros de $50 \mathrm{~mm}$ de altura y $49 \mathrm{~mm}$ de diámetro. Asímismo, para las determinaciones de pH y CE se tomó aproximadamente $500 \mathrm{~g}$ de muestra disturbada por horizonte.

\section{Determinación de la resistencia al rompi-} miento (RR) e índice de friabilidad (IF). La RR se determinó a partir de la prueba de compresión propuesta por Dexter y Watts (2001), usando una prensa mecánica CBR (Soiltext - CF410) de dos velocidades. En la prueba, los 7280 agregados, previamente pesados, se colocaron individualmente entre los dos platos paralelos del equipo y se les aplicó fuerza con una velocidad de deformación constante de $0,07 \mathrm{~mm} \mathrm{~s}^{-1}$, hasta que se produjo el rompimiento de los agregados.

El cálculo de la RR de los agregados de cada horizonte se estableció usando la ecuación propuesta por Utomo y Dexter (1981), Dexter y Kroesbergen (1985):

$$
R R=0,576\left(P / D_{e}^{2}\right)
$$

Donde, RR (generalmente expresada en $\mathrm{kPa}$ ) es la resistencia al rompimiento, $\mathrm{P}(\mathrm{N})$ el valor del pico de fuerza registrado al momento de la ruptura de cada agregado, $\mathrm{D}_{\mathrm{e}}(\mathrm{m})$ el diámetro efectivo del conjunto de agregados de cada horizonte y el valor 0,576 corresponde a una constante de proporcionalidad.

El diámetro efectivo $\left(\mathrm{D}_{\mathrm{e}}\right)(\mathrm{m})$ se calculó de acuerdo con las masas individuales de los agregados a partir de la ecuación (Dexter y Watts, 2001):

$$
D_{e}=D_{m}\left(\frac{M}{M_{0}}\right)^{1 / 3}
$$

Donde $\mathrm{D}_{\mathrm{m}}(\mathrm{m})$ es el diámetro medio de los agregados de cada horizonte, $\mathrm{M}$ (g) es la masa de cada agregado y $\mathrm{M}_{0}$ (g) la masa media del conjunto de agregados de cada horizonte. El diámetro medio de los agregados $\left(\mathrm{D}_{\mathrm{m}}\right)$ se obtuvo a partir de la ecuación propuesta por Dexter y Kroesbergen (1985):

$$
D_{m}=\left(d_{1}+d_{2}\right) / 2
$$

Donde $\mathrm{d}_{1}$ es el tamaño de abertura de malla del tamiz superior (m) y $\mathrm{d}_{2}$, el tamaño de abertura de malla del tamiz inferior $(\mathrm{m})$.

El índice de friabilidad ( $\mathrm{IF}_{\mathrm{i}}$ ) del conjunto de agregados de cada horizonte se halló a partir del coeficiente de variación propuesto por Dexter y Watts (2001), considerando el límite inferior de este coeficiente (Avila et al., 2015a):

$$
I F i=\frac{\sigma_{y}}{\bar{Y}} \pm \frac{\sigma_{y}}{\bar{Y} \sqrt{2 n}}
$$

En el que se relaciona la desviación estándar de los valores medidos de resistencia a la compresión $\left(\sigma_{y}\right)$, y las medias de la resistencia a la compresión $(\overline{\mathrm{Y}})$ medidas en $n$ réplicas; en este estudio en valor de $\mathrm{n}$ fue de 10 (agregados) por cada horizonte de suelo. El IF $F_{i}$ se interpretó con base en los parámetros establecidos por Imhoff et al. (2002).

Determinaciones físicas y químicas de los suelos. La Densidad aparente (Da) se obtuvo mediante la recolección en cada horizonte de una muestra no disturbada de suelo en un cilindro de volumen conocido (American Society of Agronomy, 1986; American Society 
of Agronomy, 2002; IGAC, 2006). El DPM y Ea se determinaron mediante la técnica de tamizado en húmedo propuesto por Yoder (1936), utilizando para ello el movimiento continuo de $100 \mathrm{~g}$ de suelo (agregados entre 6,3 y $8 \mathrm{~mm}$ ) en agua, utilizando un conjunto de tamices de tamaño de malla 6,3; 4,0; 2,$0 ; 1,0$ y $0,425 \mathrm{~mm}$ dispuestos en orden descendente, respectivamente (American Society of Agronomy, 1986; IGAC, 2006); estas dos características se hallaron mediante las relaciones:

$$
D P M=\sum_{i=1}^{n} M s s i \bar{X} i / 100
$$

Donde, DPM es el diámetro ponderado medio (mm), Mssi es la masa de la fracción de los agregados del suelo retenidos en cada tamiz (g), X $i$ es el promedio del diámetro de malla entre el tamiz superior y el subsiguiente $(\mathrm{mm})$ y 100 es la masa de agregados. El estado de agregación a partir de:

$$
E a=100-\sum M s s i
$$

En la que 100 es la masa total de agregados (g) y $\sum$ Mssi es la relación de la sumatoria de las Mss de los agregados del suelo retenidos en cada tamiz $(6,3 ; 4 ; 2 ; 1 ; 0,425 \mathrm{~mm})$.

La porosidad total se estimó a partir de la relación entre los valores de densidad real (determinada por el método del picnómetro) y la densidad aparente (determinada por el método del cilindro) (American Society of Agronomy, 1986; American Society of Agronomy, 2002; IGAC, 2006). El Ph se determinó a través del método potenciométrico en relación suelo-agua $1: 1(\mathrm{p} / \mathrm{v})$ y la conductividad eléctrica, mediante la técnica del extracto de saturación utilizando un conductímetro (American Society of Agronomy, 1996; IGAC, 2006).
Análisis estadístico. El análisis descriptivo involucró el cálculo de promedios para el índice de friabilidad en su límite inferior $\left(\mathrm{IF}_{\mathrm{i}}\right)$, diámetro ponderado medio (DPM), $\mathrm{pH}$ y conductividad eléctrica (CE) según la categoría de estabilidad del suelo y el $\mathrm{IF}_{\mathrm{i}}$ por horizonte del suelo y por la categorización del estado de agregación. Además se construyeron gráficos de superficies tridimensionales para $\mathrm{IF}_{\mathrm{i}}$ asociado con las variables Ea, DPM, Da, Pt, CE y $\mathrm{pH}$ para cada horizonte morfogenético utilizando el método de los splines para la generación de las superficies (referencias). En el componente inferencial se ajustó un modelo lineal para un diseño factorial incompleto en arreglo Completamente al Azar, para el cual se realizó un Análisis de Covarianza Multivariante (Statistical Analysis System, 2009) sobre el modelo lineal en forma matricial expresado como $\mathbf{Y}=\mathbf{X} \beta \boldsymbol{+} \mathbf{Z} \gamma+\varepsilon$, representando $\mathbf{Y}$ la matriz de respuestas asociadas al $\mathrm{IF}_{\mathrm{i}}$ en cada tamaño de agregado, $\mathbf{X}$ representó a la matriz de diseño que involucró a los factores de clasificación el horizonte y el orden taxonómico, $\beta$ representó a los parámetros asociados a los factores de clasificación, $\mathbf{Z}$ representó a la matriz de covariables (Pt y Ea), $\gamma$ representó los parámetros asociados a las covariables, y $\varepsilon$, a la matriz de errores residuales. Para estos últimos, se asumió una distribución normal multivariante con vector de medias nulo y matriz de varianzas y covarianzas $\sigma^{2} \mathbf{I}$, siendo I una matriz identidad de dimensión $\mathrm{n} \times \mathrm{n}$.

Las covariables Da y DPM no cumplieron los supuestos necesarios para permanecer en el modelo estadístico planteado. Los supuestos de normalidad multivariante e igualdad de matrices de varianzas y covarianzas se revisaron mediante la aplicación de la prueba de Roystone y la $\mathrm{M}$ de Box respectivamente (Kohl, 2015), ambas disponibles en las librerías del software libre R. 


\section{RESULTADOS Y DISCUSIÓN}

No se presentó interacción entre los factores de clasificación asociados al tipo de horizonte y la taxonomía de los suelos a nivel de orden $(\mathrm{p}=0,5888)$, tal como se observa en la Tabla 2 , con lo cual se hicieron interpretables los efectos principales, a saber, la taxonomía y el horizonte, ambos con significancias inferiores al $1 \%$, lo que condujo al rechazo de la hipotésis nula de efectos nulo en ambos casos. Esto sugiere diferencias altamente significativas en las respuestas (índices de friabilidad) como consecuencia probable del tipo de horizonte y la taxonomía considerada.

En lo que respecta a los horizontes (dos niveles), se evidencia la importancia de considerar la separación de los horizontes superficiales por sus límites naturales al momento de realizar el muestreo para determinar el $\mathrm{IF}_{\mathrm{i}}$ de los suelos, al igual que en el caso de la taxonomía, por lo que un tratamiento sin discriminación por este par de variables podría generar promedios que no representan ni al horizonte ni a la clase taxonómica considerada.

De las covariables Da y Pt, la segunda resultó significativa, lo que sugiere que tiene una relación lineal con el índice de friabilidad y podría facilitar la predicción de la friabilidad en términos de esta covariable. Autores como Watts y Dexter (1998) y Munkholm et al. $(2002 \mathrm{a}, \mathrm{b})$ han reportado una relación inversa entre friabilidad y $\mathrm{Da}$, así mismo, Larionov et al. (2017), demostraron que la

Tabla 2. Análisis de Covarianza Multivariante para los efectos considerados en el modelo lineal ajustado al diseño experimental (estadístico de prueba: Lambda de Wilks).

\begin{tabular}{|c|c|c|c|c|c|}
\hline Fuente de variación & Matriz de s & uma de & cuadrados & F calculado & $P$ valor \\
\hline Horizonte & {$\left[\begin{array}{l}0,145 \\
0,064 \\
0,104\end{array}\right.$} & $\begin{array}{l}0,064 \\
0,046 \\
0,074\end{array}$ & $\left.\begin{array}{l}0,104 \\
0,074 \\
0,120\end{array}\right]$ & 6,29 & 0,0005 \\
\hline Taxonomía (orden de suelo) & {$\left[\begin{array}{l}0,145 \\
0,074 \\
0,110\end{array}\right.$} & $\begin{array}{l}0,074 \\
0,082 \\
0,105\end{array}$ & $\left.\begin{array}{l}0,110 \\
0,105 \\
0,292\end{array}\right]$ & 3,48 & $<0,0001$ \\
\hline Horizonte*Taxonomía & {$\left[\begin{array}{l}0,014 \\
0,014 \\
0,032\end{array}\right.$} & $\begin{array}{l}0,014 \\
0,028 \\
0,027\end{array}$ & $\left.\begin{array}{l}0,032 \\
0,027 \\
0,108\end{array}\right]$ & 0,86 & 0,5888 \\
\hline Porosidad total & {$\left[\begin{array}{l}0,004 \\
0,019 \\
0,020\end{array}\right.$} & $\begin{array}{l}0,019 \\
0,096 \\
0,100\end{array}$ & $\left.\begin{array}{l}0,020 \\
0,100 \\
0,104\end{array}\right]$ & 4,61 & 0,0040 \\
\hline Estado de agregación & {$\left[\begin{array}{l}0,028 \\
0,054 \\
0,040\end{array}\right.$} & $\begin{array}{l}0,054 \\
0,106 \\
0,078\end{array}$ & $\left.\begin{array}{l}0,040 \\
0,078 \\
0,058\end{array}\right]$ & 4,33 & 0,0057 \\
\hline Error & {$\left[\begin{array}{l}1,156 \\
0,342 \\
0,337\end{array}\right.$} & $\begin{array}{l}0,342 \\
1,937 \\
0,282\end{array}$ & $\left.\begin{array}{l}0,337 \\
0,282 \\
2,422\end{array}\right]$ & - & - \\
\hline Total & {$\left[\begin{array}{l}0,280 \\
0,225 \\
0,306\end{array}\right.$} & $\begin{array}{l}0,225 \\
0,358 \\
0,384\end{array}$ & $\left.\begin{array}{l}0,306 \\
0,384 \\
0,682\end{array}\right]$ & - & - \\
\hline
\end{tabular}


RR se incrementó en los suelos con mayores valores de Da; sin embargo, en este trabajo no se encontró un efecto significativo de esta variable física sobre el $\mathrm{IF}_{\mathrm{i}}$, debido al bajo rango de variación de esta propiedad en los suelos estudiados $(1,2-1,6 \mathrm{Mg}$ $\mathrm{m}^{-3}$ ) o a las diferencias texturales. La Pt, en cambio, presentó un efecto altamente significativo $(\mathrm{p}<1 \%)$ en el índice de friabilidad (Tabla 2). Se encontró que, en general, hay un incremento de la friabilidad del suelo a mayor Pt, en especial, cuando esta presenta valores superiores al 45\% (Figura 1). Estos resultados coinciden con lo reportado por Munkholm et al. (2001) y Munkholm et al. (2012), quienes encontraron correlación positiva y significativa entre la Pt y la friabi- lidad de suelos franco limosos y franco arenosos. Es de resaltar que cuando los suelos tienen valores superiores al $45 \%$ de Pt, prevalece el efecto de esta propiedad sobre los valores altos de Da y se corresponde con los mayores valores de $\mathrm{IF}_{\mathrm{i}}$; sin embargo, a valores de Pt que están aproximadamente por debajo del 30\% y de Da inferiores a 1,3 Mg $\mathrm{m}^{-3}$, prevalece la influencia de la densidad y se presenta un incremento del valor del $\mathrm{IF}_{\mathrm{i}}$ de los suelos. En los suelos con Pt superior al 35\% y baja Da (aproximadamente $<1,3$ $\mathrm{Mg} \mathrm{m}^{-3}$ ), los valores del $\mathrm{IF}_{\mathrm{i}}$ se mantienen aproximadamente constantes. En general, los valores más altos del $\mathrm{IF}_{\mathrm{i}}$ se corresponden con los valores superiores al $45 \%$ del Pt (Figura 1).

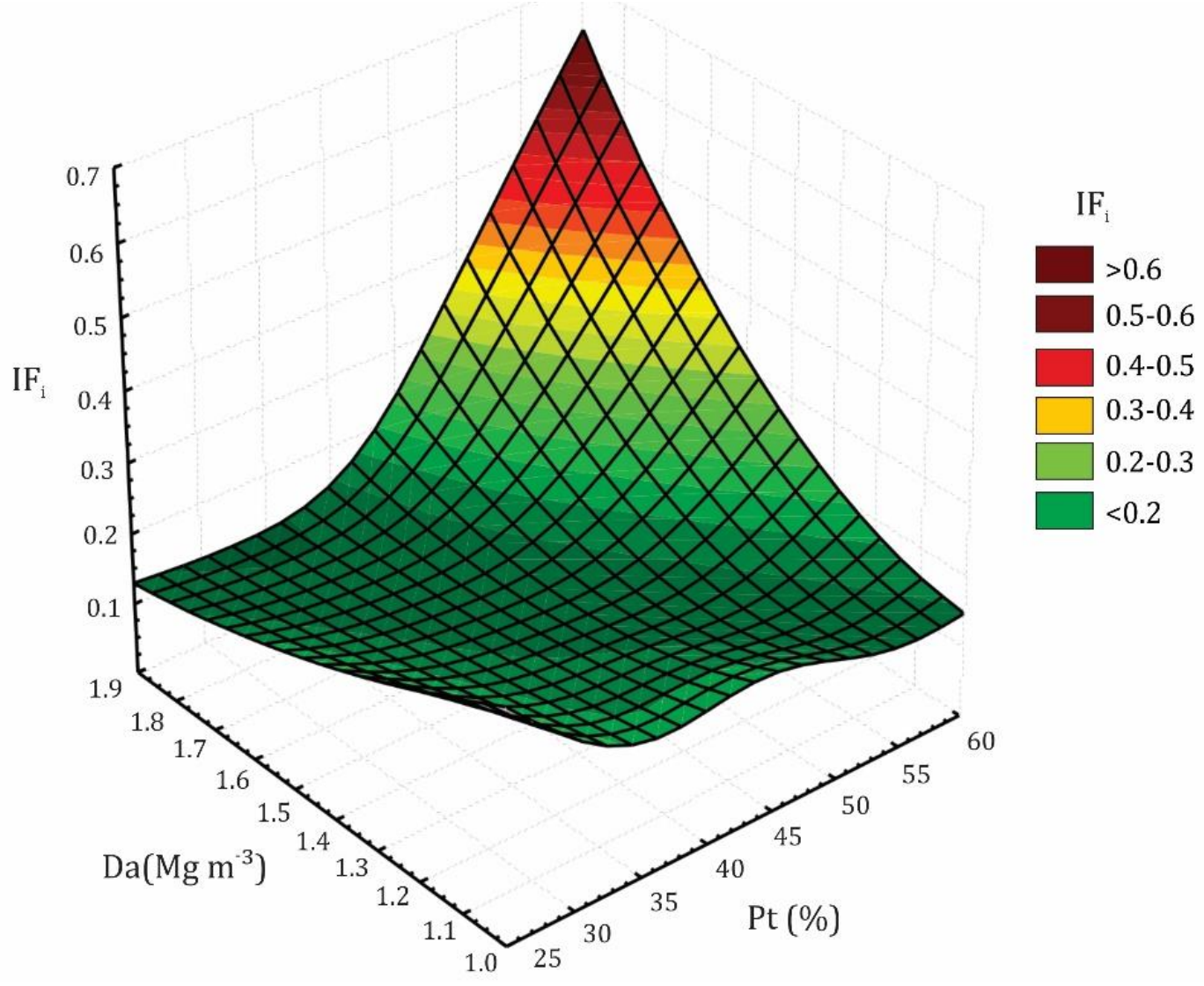

Figura 1. Relación de las variables densidad aparente (Da), porosidad total (Pt) e índice de friabilidad en su límite inferior $\left(\mathrm{IF}_{\mathrm{i}}\right)$, por el método spline. 
Para Dexter y Watts (2001) el grado de desarrollo estructural y la estabilidad de los agregados afectan la friabilidad. En el presente estudio se evaluó el efecto de la estabilidad de la estructura, específicamente el DPM (diámetro ponderado medio de los agregados retenidos en los tamices luego del humedecimiento) y el Ea (porcentaje de agregados de tamaño superior a $0,425 \mathrm{~mm}$ luego del humedecimiento) en el $\mathrm{IF}_{\mathrm{i}}$ de los suelos. Algunos autores (Munkholm, 2011), sostienen que la cama de siembra óptima se obtiene con agregados de tamaños entre 0,5 y $8 \mathrm{~mm}$, los cuales idealmente deben tener alta RR (baja friabilidad), mientras que otros investigadores (Tisdall y Adem, 1986), sugieren que los agregados ideales son aquellos que están entre 1 y $10 \mathrm{~mm}$ $\mathrm{y}$ son estables al humedecimiento. El DPM no mostró efecto significativo sobre la friabilidad con el modelo estadístico utilizado; contrario a esto, el Ea, presentó efecto altamente significativo $(\mathrm{p}<1 \%)$ (Tabla 2). Aunque de manera inferencial no se encontró significancia en el efecto del DPM sobre el IF ${ }_{i}$.

En la Tabla 3 se presenta de manera descriptiva el comportamiento de estas dos propiedades, donde se observa como en el horizonte superficial (Ap) el $\mathrm{IF}_{\mathrm{i}}$ tiende a incrementarse en la medida que se hace mayor el DPM (suelos más estables) en correspondencia con el decrecimiento de los valores de $\mathrm{pH}$ y CE; mientras que en el horizonte subsuperficial (A1) no se observó una tendencia clara del $\mathrm{IF}_{\mathrm{i}}$ en relación con la estabilidad estructural de los suelos, aunque se mantiene la tendencia decreciente del pH y la CE a medida que los agregados se hacen más estables. Por su parte, el $\mathrm{IF}_{\mathrm{i}}$ se incrementó en la medida en que los suelos presentaron un mayor Ea, excepto cuando este último fue menor del 25\% (muy bajo estado de agregación) (Tabla 4).

Tabla 3. Índice de friabilidad en su límite inferior $\left(\mathrm{IF}_{\mathrm{i}}\right)$, diámetro ponderado medio (DPM), pH y conductividad eléctrica (CE) con relación a la estabilidad de los suelos bajo estudio.

\begin{tabular}{clcccc}
\hline Horizonte & Estabilidad del suelo* & $\mathbf{I F}_{\mathbf{i}}$ & $\begin{array}{c}\mathbf{D P M} \\
\mathbf{( \mathbf { m m } )}\end{array}$ & $\mathbf{p H}$ & $\begin{array}{c}\mathbf{C E} \\
\mathbf{m S ~ c m}^{-\mathbf{1}}\end{array}$ \\
\hline \multirow{3}{*}{$\mathrm{Ap}$} & Inestable & 0,37 & 0,41 & 6,80 & 1,60 \\
& Ligeramente estable & 0,38 & 1,01 & 6,70 & 1,10 \\
& Moderadamente estable & 0,39 & 2,15 & 6,40 & 0,80 \\
& Estable & 0,42 & 3,70 & 5,50 & 0,60 \\
\hline \multirow{3}{*}{$\mathrm{A} 1$} & Inestable & 0,38 & 0,41 & 7,10 & 1,20 \\
& Ligeramente estable & 0,35 & 0,92 & 6,80 & 1,10 \\
& Moderadamente estable & 0,34 & 2,24 & 6,20 & 0,60 \\
& Estable & 0,35 & 3,93 & 5,50 & 0,60 \\
\hline
\end{tabular}

*IGAC, 2006. 
Tabla 4. Estado de agregación (Ea) e Índice de friabilidad en su límite inferior (IFi) de los suelos estudiados (horizontes Ap y A1).

\begin{tabular}{cccc}
\hline Ea (\%) & $\begin{array}{c}\mathbf{I F}_{\mathbf{i}} \\
\mathbf{A p}\end{array}$ & $\begin{array}{c}\mathbf{I F}_{\mathbf{i}} \\
\mathbf{A 1}\end{array}$ & $\begin{array}{c}\mathbf{I F}_{\mathbf{i}} \\
\mathbf{A p}-\mathbf{A} \mathbf{1}\end{array}$ \\
\hline$<25$ & 0,38 & 0,33 & 0,36 \\
$25-35$ & 0,38 & 0,32 & 0,35 \\
$35-80$ & 0,39 & 0,35 & 0,37 \\
$80-90$ & 0,39 & 0,37 & 0,38 \\
$>90$ & 0,45 & 0,35 & 0,40 \\
\hline
\end{tabular}

El análisis del comportamiento del $\mathrm{IF}_{\mathrm{i}} \mathrm{a}$ partir de la interacción del DPM y el Ea de los suelos (Figura 2), muestra como en los valores bajos de DPM $(<1,5 \mathrm{~mm})$, a medida que los suelos presentaron un mayor Ea, el $\mathrm{IF}_{\mathrm{i}}$ tiene una tendencia al incremento; sin embargo, en valores de Ea menores al 25\% (muy bajos) el IF se incrementó en la medida en que el DPM fue mayor. Cuando el Ea fue superior al 25\% (bajos, medios y altos) el $\mathrm{IF}_{\mathrm{i}}$ mostró tendencia al decrecimiento con el incremento del DPM.

También se evaluó el efecto de algunas características químicas de los suelos sobre la friabilidad, específicamente se consideraron el pH y la CE. El efecto del carbono orgánico como propiedad química clave en el índice de friabilidad fue evaluado en un trabajo previo (Avila et al., 2015b). De acuerdo con Munkholm (2011) y Kim et al. (2007) se espera que los bajos valores de pHy saturación de bases provoquen altas resistencias en los suelos en condición seca; sin embargo, en este trabajo no se encontró significancia en el efecto del $\mathrm{pH}$ y la CE en el $\mathrm{IF}_{\mathrm{i}}$ de los suelos.

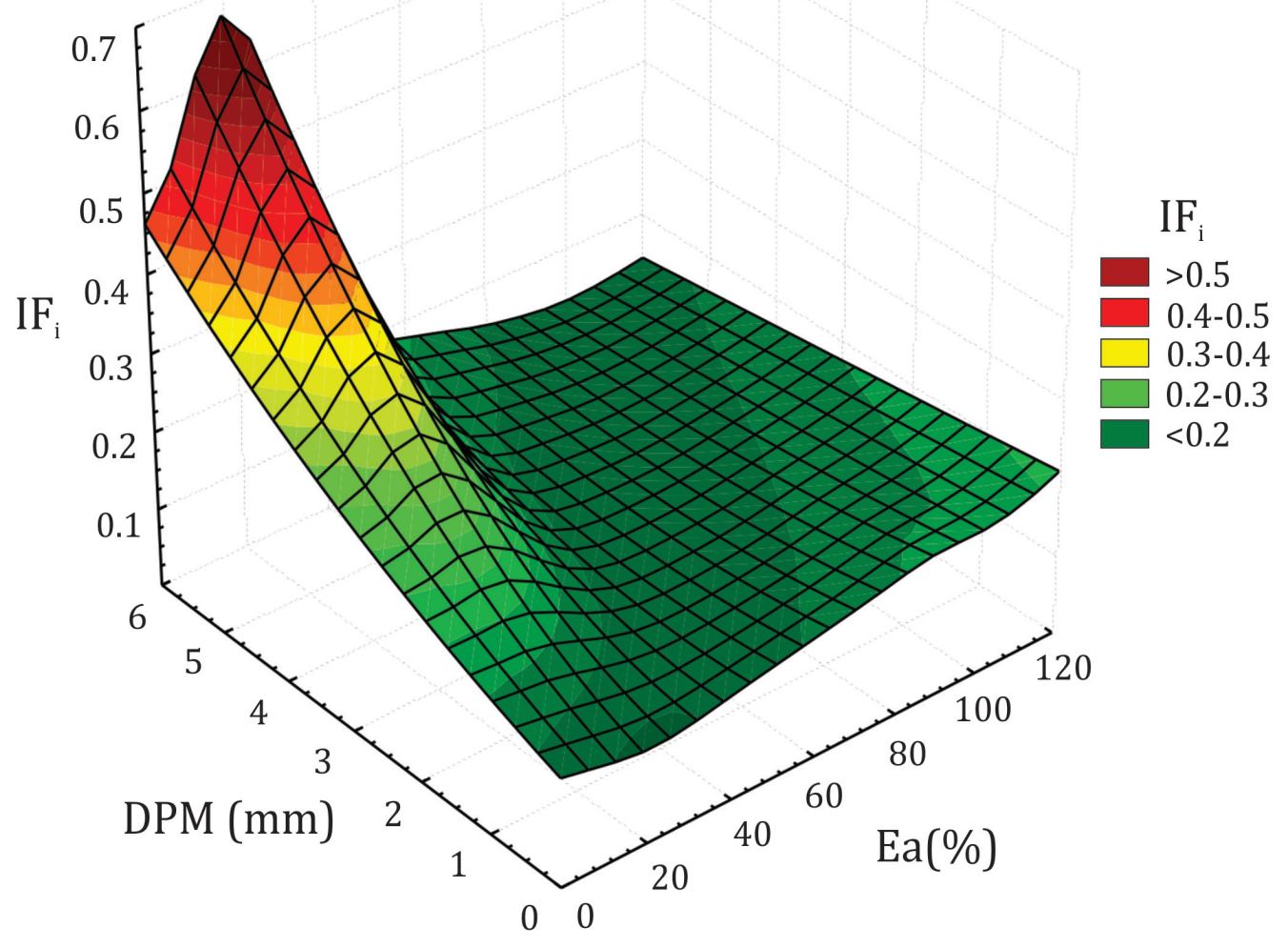

Figura 2. Relación de las variables diámetro ponderado medio (DPM), estado de agregación (Ea) e índice de friabilidad en su límite inferior $\left(\mathrm{IF}_{\mathrm{i}}\right)$, obtenida por el método spline. 
Del análisis descriptivo se encontró que, en general, en el horizonte Ap los valores de $\mathrm{IF}_{\mathrm{i}}$ se comportaron de manera inversa a los de CE y $\mathrm{pH}$ en la medida que los suelos adquirieron mayor estabilidad (Tabla 3), lo cual coincide con lo reportado por Rahimi et al. (2000), quienes encontraron que los suelos con mayor CE presentaron a su vez las más altas $\mathrm{RR}$, lo que podría interpretarse como una relación inversa entre $\mathrm{CE}$ e $\mathrm{IF}_{\mathrm{i}}$. Esta relación no se observó en el horizonte A1, donde el $\mathrm{IF}_{\mathrm{i}}$ no mostró una tendencia clara en relación con el pH, la CE y la estabilidad de los suelos.

\section{CONCLUSIONES}

Los resultados de esta investigación indican que propiedades físicas como la estabilidad de agregados y la porosidad total influyen en el comportamiento del índice de friabilidad de los suelos. Por el contrario, la densidad aparente y el diámetro ponderado medio de los agregados no mostraron tener efecto sobre la variación del índice de friabilidad.

Propiedades químicas como el pH y la conductividad eléctrica no mostraron relación directa con el índice de friabilidad, debido probablemente a los rangos bajos de variación de estos parámetros. Por lo tanto, se sugiere realizar nuevas investigaciones utilizando suelos en los que el rango de variación de estas propiedades sea mayor.

\section{AGRADECIMIENTOS}

A la Universidad del Tolima, a COLCIENCIAS y a la Dirección de Investigaciones de la Universidad Nacional de Colombia por el apoyo económico; a los agricultores que permitie- ron en sus predios el muestreo de suelos; a CENICAÑA por el aporte de información y a los laboratorios de suelos de la Universidad Nacional de Colombia y el Instituto Geográfico Agustín Codazzi por la colaboración en la fase analítica de la investigación.

\section{REFERENCIAS BIBLIOGRÁFICAS}

1. Alaoui, A.; Lipiec, J.; Gerke, H.H. 2011. A review of the changes in the soil pore system due to soil deformation: a hydrodynamic perspective. Soil Till. Res.115 - 116: 1 - 15. doi:10.1016/j.still.2011.06.00

2. American Society Of Agronomy. 2002. Methods of Soil Analysis. Part 4: Physical Methods. Jacob Dane, Editor. Soil Science Society of America, Inc. Publisher Madinson, Wisconsin USA, 1692p.

3. American Society of Agronomy. 1986. methods of soil analysis. Part 1: Physical and Mineralogical Methods. Second Edition. Arnold Klute, Editor. Soil Science Society of America, Inc. Publisher Madinson, Wisconsin USA, 1188p.

4. American Society Of Agronomy. 1996. Methods of Soil Analysis. Part 3: Chemical Methods J. M Bartels, Editor. Soil Science Society of America, Inc. Publisher Madinson, Wisconsin USA, 1390p.

5. Avila, E.A.; Leiva, F.R.; Darghan, E.; Madriñán. R. 2015a. Effect of aggregate size and superficial horizon differentiation on the friability index of soils cultivated with sugar cane: a multivariate approach. Agronomía Colombiana. 33(1):92 - 98. doi: 10.15446/agron. colomb.v33n1.49855.

6. Avila, E.A.; Leiva, F.R.; Madriñán. R. 2015b. Friability and its relationship with clay and organic carbon in soils cultivated with sugar cane. Agronomía Colombiana. 33(2):365 - 372. doi:10.15446/agron.colomb.33(3):54068. 
7. Braunack, M.V.; Hewitt, J.S.; Dexter. A.R. 1979. Brittle fracture of soil aggregates and the compaction of aggregate beds. J. Soil Sci. 30(4):653 - 667. doi: 10.1111/j.13652389.1979.tb01015.x.

8. Dexter, A.R.; Kroesbergen, B. 1985. Methodology for determination of tensile strength of soil aggregates. J. Agr. Eng. Res. 31(2):139 147. doi: 10.1016/0021-8634(85)90066-6.

9. Dexter, A.R.; Watts. C.W. 2001. Tensile strength and friability. In: Smith, K.A., Mullins, C.E. (Eds.), Soil and Environmental Analysis: Physical Methods. Segunda edición. Marcel Dekker Inc, New York. 405 - 433 p.

10. Esengun, K., G.; Erdal, O.; Gunduz; Erdal. H. 2007. An economic analysis And energy use in stake-tomato production in Tokat province of Turkey. Renew Ener. 32(11):1873 1881. doi: 10.1016/j.renene.2006.07.005.

11. Etana, A.; Larsbo, M.; Keller, T.; Arvidsson, J.; Schjønning, P.; Forkman,; Jarvis, N. 2013. Persistent subsoil compaction and its effects on preferential flow patterns in a loamy till soil. Geoderma. 192:430 - 436. doi: 10.1016/j. geoderma.2012.08.015.

12. Guimarães, R.M.; Tormena C.A.; Alves S.J.; Fidalski, J.; Blainski. E. 2009. Tensile strength, friability and organic carbon in an oxisol under a crop-livestock system. Scientia Agricola. (Piracicaba, Brasil.). 66(4):499 - 505. doi:10.1590/S0103-90162009000400011.

13. IGAC y CENICAÑA. Instituto Geográfico Agustín Codazzi y Centro de Investigación de la Caña de Azúcar de Colombia. 2006. Estudio detallado de suelos y capacidad de uso de las tierras sembradas con caña de azúcar en el Valle Geográfico del Río Cauca. Informe General, Cali, 62 p. En: http: //www.cenicana. org/publicaciones/publicaciones.; consulta: febrero, 2017.

14. IGAC. Instituto Geográfico Agustín Codazzi. 2006. Métodos analíticos del laboratorio de suelos. Sexta edición. Bogotá. 674 p.
15. IGAC. Instituto Geográfico Agustín Codazzi. 1977. Zonas de vida o formaciones vegetales de Colombia. Memoria explicativa sobre el mapa ecológico. Bogotá: IGAC. 238p.

16. Imhoff, S., Da Silva, P.A.; Dexter. A.R. 2002. Factors contributing to the tensile strength and friability of Oxisols. Soil Sci. Soc. Am. J. 66:1656-1661. doi: 10.2136/ sssaj2002.1656.

17. Kim, T.H.; Kim, C.K.; Jung, S.J.; J.H. Lee. 2007. Tensile strength characteristics of contaminated and compacted sand-bentonite mixtures. Environ. Geol. 52:653 - 661. doi: 10.1007/s00254-006-0494-8.

18. Kohl, M. 2015. Introduction to statistical data analysis with R. First edition. Bookboon. London. 228p.

19. Kuncoro, P.H.; Koga, K.; Satta, N.; Muto. Y.2014. A study on the effect of compaction on transport properties of soil gas and water I: Relative gas diffusivity, air permeability, and saturated hydraulic conductivity. Soil Till. Res. 143:172 - 179. doi: 10.1016/j. still.2014.02.006.

20. Larionov, G.A.; Dobrovol'skaya, N. G.; Kiryukhina, Z. P.; Krasnov, S. F.; Litvin, L. F.; Gorobets, A. V.; Sudnitsyn. I. I. 2017. Effect of Soil Density, Tensile Strength, and Water Infiltration on the Rupture Rate of Interaggregate Bonds. Eurasian Soil Science. 50(3):335 - 340. doi: 10.1134/S1064229317010094.

21. Macks, S.P., Murphy, B.W. Cresswell, H.P.; Koen. T.B. 1996. Soil friability in relation to management history and suitability for direct drilling. Aust. J. Soil Res. 34:343 - 360. doi: 10.1071/SR9960343.

22. Munkholm, L. J. 2011. Soil friability: A review of the concept, assessment and effects of soil properties and management (Review). Geoderma. 167-168:236 - 246. doi: http: 10.1016/j.geoderma.2011.08.005. 
23. Munkholm, L.J.; Heck, R.J.; Deen. B. 2012. Soil pore characteristics assessed from X-ray micro-CT derived images and correlations to soil friability. Geoderma. 181 - 182:22 - 29. doi:10.1016/j.geoderma.2012.02.024.

24. Munkholm, L.J.; Heck R.J.; Deen B.; T. Zidar. 2016. Relationship between soil aggregate strength, shape and porosity for soils under different long-term management. Geoderma. 268:52 - 59. doi: 10.1016/j.geoderma.2016.01.005.

25. Munkholm, L.J.; Schjønning, P.; Kay. B.D. 2002a. Tensile strength of soil cores in relation to aggregate strength, soil fragmentation and pore characteristics. Soil Till. Res. 64(1-2):125 - 135. doi: 10.1016/S01671987(01)00250-1.

26. Munkholm, L.J.; Schjønning, P.; Debosz, K.; Jensen, H.E.; Christensen. B.T. 2002b. Aggregate strength and mechanical behaviour of a sandy loam soil under long-term fertilization treatments. Eur. J. Soil Sci. 53:129 - 137. doi: 10.1046/j.1365-2389.2002.00424.x.

27. Munkholm, L.J.; Schjønning, P.; Petersen. C.T. 2001. Soil mechanical behavior of sandy loams in a temperate climate: case studies on long-term effects of fertilization and crop rotation. Soil Use Manage. 17:269 - 277. doi: 10.1111/j.1475-2743.2001.tb00037.x.

28. Rahimi, H.; Pazira, E.; Tajik. F. 2000. Effect of soil organic matter, electrical conductivity and sodium adsorption ratio on tensile strength of aggregates. Soil Till. Res. 54:145 153. doi: 10.1016/S0167-1987(00)00086-6.

29. Reis, D.; Rodriguez, C.; Pauletto, E.; Dupont, P.; Pillon, C. 2014. Tensile strength and friability of an Alfi sol under agricultural management systems. Sci. Agric. 71(2):163 - 168. doi: 10.1590/S0103-90162014000200012.

30. Schjǿnning, P.; De Jonge, L.W.; Munkholm, L.J.; Moldrup, P.; Christensen, B.T.; Olesen. J.E. 2012. Clay dispersibility and soil friability - testing the soil clay-to-carbon saturation concept. Vadose Zone J. 11(1). doi: 10.2136/ vzj2011.0067.
31. Sefeedpari, P.; Shokoohi, Z;; Behzadifar. Y. 2014. Energy use and carbon dioxide emission analysis in sugarcane farms: a survey on Haft-Tappeh Sugarcane Agro-Industrial Company in Iran. Journal of Cleaner Production. 83:212 - 219. doi: 10.1016/j.jclepro.2014.07.048.

32. SAS - Statistical Analysis System. 2009. SAS users guide; SAS/STAT - 9.3 (1) User's guide the GLM procedure (Book excerpt), SAS Campus Drive, Cary North Carolina Rv. 27513, $206 \mathrm{p}$.

33. Tisdall, J.M.; Adem, H.H. 1986. Effect of water content of soil at tillage on size distribution of aggregates and infiltration. Australian Journal of Experimental Agriculture. 26:193 - 195. doi: 10.1071/EA9860193.

34. USDA - United States Department of Agriculture. 2010. Keys to Soil Taxonomy, Eleventh Edition. By Soil Survey Staff. USDA-NRCS, Washington, D.C., USA. 365p.

35. Utomo, W.H.; Dexter. A.R. 1981. Soil friability. J. Soil Sci. 32:203 - 213. doi: 10.1111/j.13652389.1981.tb01700.x.

36. Watts, C.W.; Dexter. A.R. 1998. Soil friability: theory, measurement and the effects of management and organic carbon content. Eur. J. Soil Sci. 49(1):73 - 84. doi: 10.1046/j.13652389.1998.00129.x.

37. Ye, C.; Gou, Z.; Cai, C.; Wang, J.; Deng, J. 2017. Effect of water content, bulk density, and aggregate size on mechanical characteristics of Aquults soil blocks and aggregates from subtropical China. J Soils Sediments 17(1):210 219. doi: $10.1007 / s 11368-016-1480-8$.

38. Yoder, R.E., 1936. A direct method of aggregates analysis and a study the physical nature of erosion losses. J. Ame. Soc. Agron. 28(1):337 - 351. 